\title{
Death à la carte
}

\section{The 8th European Workshop on Cell Death; June 3-8, 2012 Monêtier-les-Bains, Serre Chevalier Valley, France}

\author{
Thomas R. Jackson, ${ }^{\dagger}$ Samantha E.T. Larkin, ${ }^{\dagger}$ Olubukunola A. Pickard ${ }^{\dagger}$ and Paul A. Townsend* \\ Cancer Sciences Unit; Cancer Research UK Centre; University of Southampton; Southampton, UK
}

'These authors contributed equally to this work.

Keywords: European Workshop on Cell Death, cell death, Bcl-2 family, death receptor signaling, pattern-recognition receptors, TNF, inflammation, autophagy, metabolism

Delegates arrived at the hotel on a rainy Sunday evening where they were immediately made welcome and encouraged to relax over dinner while chatting to other EWCD (European Workshop on Cell Death) participants. The EWCD is a bi-annual conference that focuses on all aspects of cell death. Each conference is held in a different unique location, with this year's being no exception. The six-day meeting took place in the Serre Chevalier Valley, France, in the picturesque village of Monêtierles-Bains. Over the course of the week there were sessions on the Bcl-2 family, death receptor signaling, pattern-recognition receptors and IAPs, XIAP and TNF, inflammation, autophagy, cell death and metabolism, mechanistic insights into tumorigenesis and tumor therapy.

The conference opened with the Bcl-2 family session, chaired and introduced by Paul Ekert (Walter and Eliza Hall Institute). After introducing the session, he went on to discuss his work on the molecular mechanisms by which HoxA9 and HoxB8 overexpression can cause leukemia, in a Bcl-2-dependent and -independent manner respectively. After lunch, Conor Kearney, from Seamus Martin's lab in Dublin, presented his work on TNF-induced cytokine production by inhibitors of apoptosis (IAPs). He presented his study of the immune consequences of small molecule IAP antagonists such as BV6. Previous studies found that IAP antagonism sensitizes tumor cells to TNF-induced apoptosis. Conor found that IAP antagonism also results in a dramatic modulation of many pro-inflammatory mediators, highlighting their possible role as regulators of inflammation. Later, Stephen Tait from the Beatson Institute, presented an exciting talk on RIP3K-mediated necroptosis. He used the E3 ubiquitin ligase, Parkin, to deplete cells of mitochondria through mitophagy. As expected, these mitochondrial depleted cells were not able to undergo apoptosis, but interestingly there was no clear inhibition of TNF-induced necroptosis. The day ended with the first of two poster sessions encompassing a wide range of cell death signaling topics.

${ }^{*}$ Correspondence to: Paul A. Townsend; Email: p.a.townsend@soton.ac.uk Submitted: 07/03/12; Accepted: 07/04/12

http://dx.doi.org/10.4161/jkst.21369
Seamus Martin began the next day with a session on inflammation. Seamus's talk challenged the generally accepted paradigm that apoptosis is not pro-inflammatory, and is therefore "good," whereas necroptosis is pro-inflammatory, so is thought to be "bad." Seamus found that Fas/TRAIL/TNF stimulated apoptosis resulted in pro-inflammatory cytokine and chemokine (IL-6, CRCX1) production. Active discussion was stimulated by Yang Liu's (University of Texas Southwestern Medical Center) presentation on the stimulation of autophagic cell death by an evolutionary conserved Beclin-1 peptide. Liu found that the Beclin-1 peptide (Tat-Beclin-1) was a potent inducer of autophagy, which was accompanied by cell death in the absence of caspase activation and was not rescued by apoptosis or necrosis inhibitors - a surprising observation that led to a lively debate. After lunch, Jennifer Martinez (St. Jude Children's Research Hospital) presented her work on LC3-associated phagocytosis and IFN- $\alpha$ secretion. She demonstrated that stimulation of the autophagic and phagocytic pathways by DNA-containing immune complexes is necessary for TLR9-mediated production of IFN- $\alpha$. The mechanism by which this occurs does not utilize the conventional autophagic pre-initiation complex. This work suggests a novel role for autophagy in response to inflammation. In the evening, the second of the poster sessions was held, which largely covered cell death in the context of therapy and tumorigenesis.

The morning of the following day focused on new mechanistic insights into tumorigenesis. Sarah Pensa (University of Cambridge) spoke on the role of the JAK-STAT and PI3K pathways in mammary gland involution. Previous work had shown that STAT3 is required for non-classical lysosomalmediated cell death. Sarah reported the use of a microarray approach to identified two PI3K regulatory subunits, p50 $\alpha$ and $\mathrm{p} 55 \alpha$, that were also STAT3 transcriptional targets. Mouse knockdowns demonstrated the importance of these subunits in mammary gland involution. Further, deregulation of Cathepsin $\mathrm{B}$ and $\mathrm{L}$ suggested a role of $\mathrm{p} 50 \alpha / \mathrm{p} 55 \alpha$ in lysosomal-mediated cell death. Mikkel Rohde (Danish Cancer Society Research Center) previously identified siramesine as an inducer of lysosomal cell death in cancer. Here, he presented recent data 


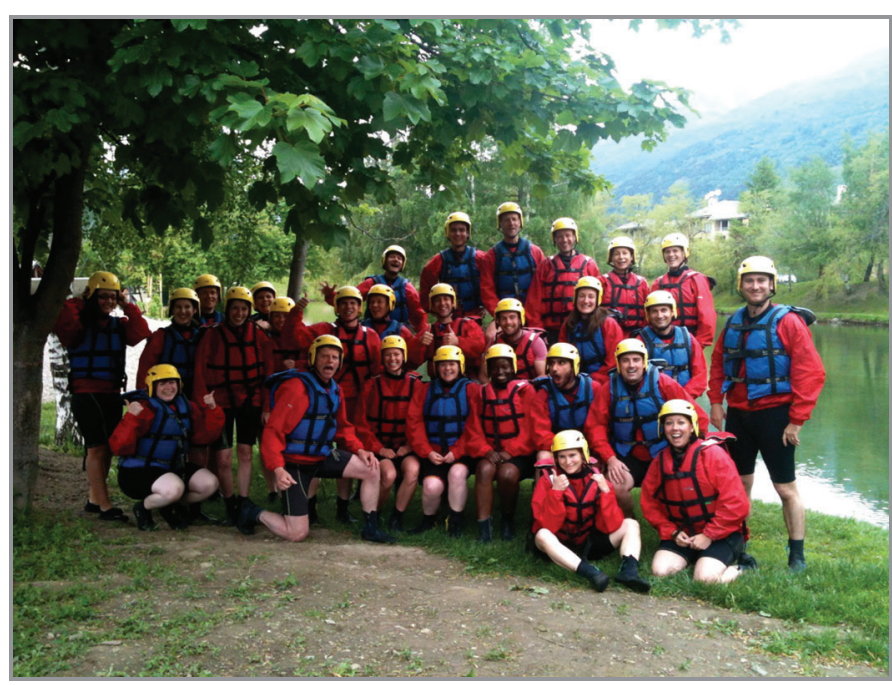

Figure 1. The whitewater rafting expedition.

identifying acid sphingomyelinase (ASM) as the target of siramesine, possibly explaining the cancer cell selectivity of siramesine as ASM is differentially expressed between cancer and normal cells. Siramesine is already approved for the treatment of anxiety, which gives these findings, once validated, great clinical relevance.

After lunch, the delegates had the opportunity to partake in one of several activities: white water rafting, mountain biking or hiking. We only took part in the rafting and biking so we cannot comment on the hiking, although Paul Ekert enjoyed himself immensely as he was the only male in the hiking troop! The rafters (Fig. 1) were taken to a neighboring village to be fitted for wetsuits and life jackets before being taken $8 \mathrm{~km}$ up-river. Within the first few minutes of paddling down the Guisanne River, rafters were instructed to paddle straight toward a giant boulder. After recovering from this, the rafts meandered down a relatively calm river interspersed with a few sections of exhilarating rapids.

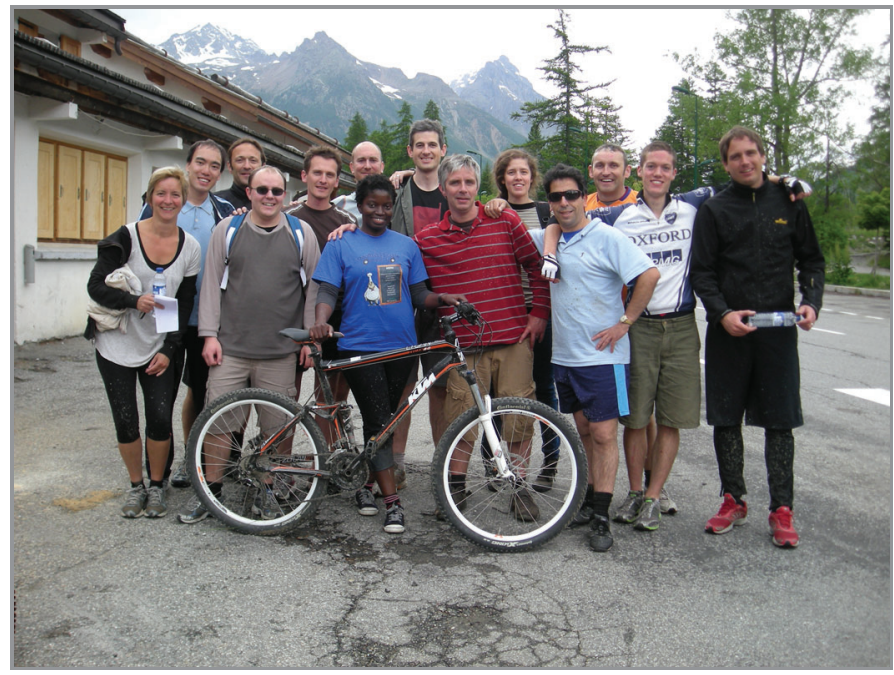

Figure 2. The mountain biking expedition.
Rafting was a resounding success... all participants returned relatively unscathed... however, Vincenzo De Laurenzi did end up falling in!

Following an ear-popping uphill half-hour drive, the mountain bikers (Fig. 2) were greeted by a snowy vista. The mountain bikers began their descent with the advice to avoid using their brakes, but not all of the mountain bikers heeded this advice, with at least one "cyclist" (Anastasis Stephanou) taking a tumble. The route included several rocky paths, a few stream crossings and some quaint villages.

That evening, delegates were taken for a beautiful dinner overlooking the mountains and old town of nearby Briançon.

The final day continued the theme of new mechanistic insights into tumorigenesis and also covered tumor therapy. Ricky Johnstone (Peter MacCallum Cancer Centre) presented his paradigm-shifting work on histone deacetylases (HDACs) in tumor development. His thought provoking research showed that inducible knockdown of HDAC3 in $\mathrm{E} \mu$-myc lymphomas resulted in their disappearance from culture, whereas knockdown of HDACs 1 and 2 increased proliferation and/or survival. Currently, pan-specific HDAC inhibitors are used to treat hematological malignancies; this work suggests that this may not be ideal and highlights the specific HDAC that may be the key to improved therapeutics using these molecules. Markus Rehm (Royal College of Surgeons in Ireland) presented his systems biology approach to studying cell death responsiveness in melanoma cell lines, a clearly state-of-the-art program where he measured the basal concentrations of apoptosis regulators and was able to accurately model and predict responsiveness to DTIC, TRAIL and cisplatin. This presentation highlighted the utility of bioinformatics and systems-level analysis for studying therapeutic response. Eric Eldering (Academic Medical Center) had the privilege of giving the final talk of the meeting, covering his work on the roles of $\mathrm{Abl}$ and $\mathrm{Btk}$ in dasatinib resistance. Following the identification of dasatinib targets in response to CD40 triggering in CLL (chronic lymphocytic leukemia), functional studies were

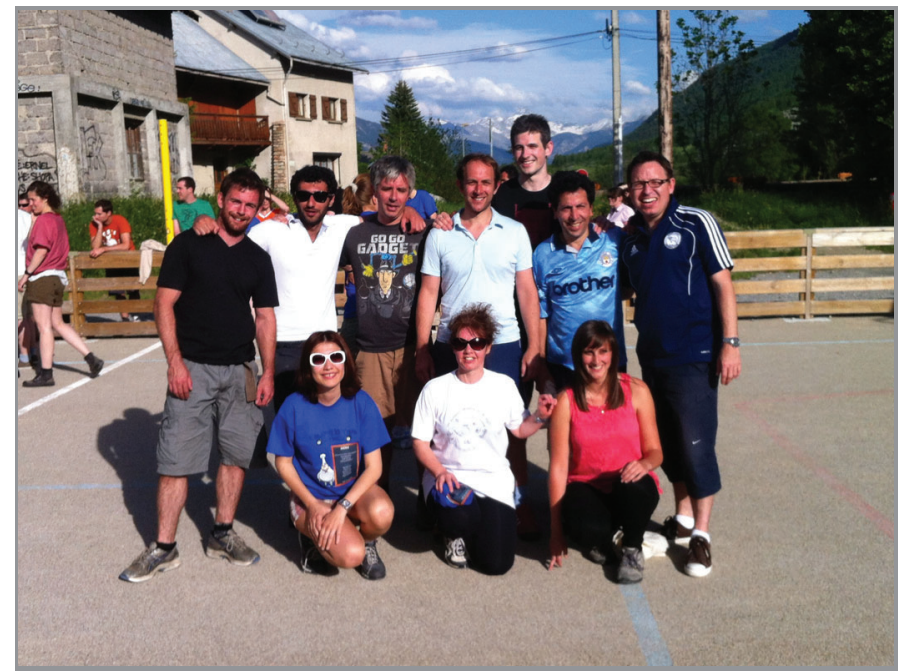

Figure 3. "The Commonwealth," "captained" by Paul Townsend. 
performed. Eric found that, in lymph nodes, Abl was involved in drug resistance and Btk aided cell adhesion and migration in CLL attachment.

The final day ended with the hastily organized EWCD football competition by Paul Townsend and Anastasis Stephanou followed by a party. Reigning champions, England, "captained" rather chaotically by Paul, reformed their team to become "The Commonwealth" (Fig. 3) and were (sadly) defeated $2-0$ by the Dutch. This bitter disappointment ensured they were well practiced for Euro 2012! The meeting was full of opportunities to mingle with like-minded researchers and the final evening offered us the chance to party with our potential new collaborators. A great time was had by all with the party continuing until the following morning, which resulted in some sore heads and interesting airport transfers... Finally, the organizers should be thanked for arranging everything at the EWCD, with special thanks to the refreshment team, who provided amazing homemade cakes and drinks between the sessions. 